\title{
General recognition theory of categorization: A MATLAB toolbox
}

\author{
LEOLA A. ALFONSO-REESE \\ San Diego State University, San Diego, California
}

\begin{abstract}
General recognition theory (GRT) is both a theory of categorization and a framework for studying human categorization behavior. The GRT toolbox is a set of MATLAB scripts and subroutines that can help an experimenter design categorization experiments, generate stimuli for these experiments, simulate a participant's responses, analyze categorization data, and graph results. The typical user designs experiments for two-category tasks in which the categories are specified by multivariate normal distributions. The toolbox also provides tools for fitting the general linear classifier and the general quadratic classifier to a data set.
\end{abstract}

General recognition theory (GRT; Ashby, 1992), also known as decision-bound theory, is a multidimensional version of signal detection theory (Green \& Swets, 1966; Thurstone, 1927). It was developed by psychologists (Ashby \& Gott, 1988; Ashby \& Townsend, 1986) to study human categorization and identification. Although portions of the GRT toolbox may aid the study of identification, the toolbox focuses primarily on categorization problems. ${ }^{1}$ In this paper I describe a typical categorization experiment, discuss assumptions of GRT in the context of a specific categorization task, and describe how the GRT toolbox may aid research on human categorization.

\section{Typical Categorization Experiment}

In a typical categorization experiment, the participant learns to sort a series of stimuli into two or more categories. The stimuli are often geometric shapes such as lines varying in length and orientation (as in Ashby, AlfonsoReese, Turken, \& Waldron, 1998), color patches varying in saturation and brightness (as in Maddox \& Dodd, 2003; Nosofsky \& Palmeri, 1997), circles with spokes projecting from the center (as in Ashby \& Maddox, 1990; Thomas, 1998), or bar patterns varying in height (as in AlfonsoReese, Ashby, \& Brainard, 2002). On each trial, the observer views a stimulus, assigns the stimulus to a category by selecting an available category label (e.g., "dog" or "cat"), and receives corrective feedback. Over the course of many trials, the observer learns to assign the correct categorization response to the majority of the stimuli.

In order to study the processes involved in human categorization, the experimenter must cleverly design the

Correspondence concerning this article should be addressed to L. A. Alfonso-Reese, Department of Psychology, San Diego State University, 5500 Campanile Drive, San Diego, CA $92182-4611$ (e-mail: leola@ alum.mit.edu). stimulus set by controlling factors such as the category structure and the prior probability of each category. Specifically, the experimenter may want to control the extent to which two categories overlap, the number of stimuli sampled from each category, or the distance between the category prototypes. GRT provides the experimenter with the mathematical framework necessary to control such factors.

\section{GRT Assumptions and a Categorization Task}

According to GRT, a stimulus may be represented as a point in multidimensional space. For example, the stimulus in Figure 1 may be represented by the point $(210,60)$, where 210 specifies the diameter of the circle and 60 specifies the clockwise degrees of rotation from 12 o'clock. When a stimulus is repeatedly presented across trials or the observer gets multiple looks at a stimulus of long duration, the result is a distribution of perceptual effects, rather than a single perceptual effect. Thus, the perceptual effects of a single multidimensional stimulus are best represented by a multidimensional probability distribution in perceptual space, usually a multivariate normal. In turn, a category of many stimuli is represented by a mixture of the associated stimulus distributions, also a multivariate normal. In order to classify the stimuli, GRT assumes that the perceptual space is divided into regions, which are separated by decision boundaries and assigned category labels. When observers perceive a stimulus as falling within a particular region, they respond with the corresponding category label. When the categories are defined by multivariate normal distributions, the optimal division of the perceptual space is a linear or quadratic decision boundary (see, e.g., Ashby, 1992; Johnson \& Wichern, 2002).

To make these assumptions more concrete, consider two categories, $\mathrm{A}$ and $\mathrm{B}$, which are each represented by 


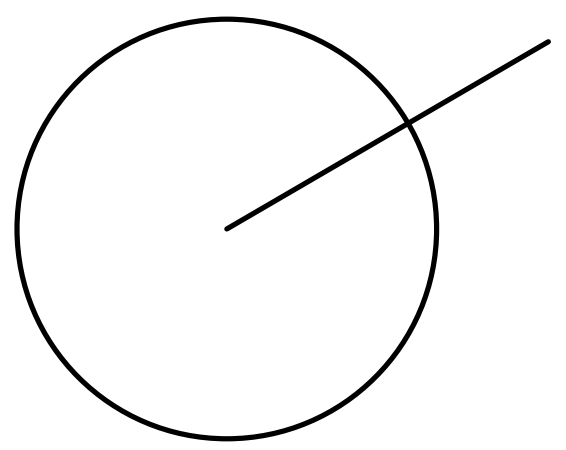

Figure 1. A two-dimensional circle-spoke stimulus represented by the point $(210,60)$.

a bivariate normal distribution. Suppose the means and covariances that define these distributions are

$$
\begin{aligned}
& \mu_{\mathrm{A}}=\left[\begin{array}{l}
172 \\
195
\end{array}\right], \\
& \mu_{\mathrm{B}}=\left[\begin{array}{l}
228 \\
125
\end{array}\right],
\end{aligned}
$$

and

$$
\Sigma_{\mathrm{A}}=\Sigma_{\mathrm{B}}=\left[\begin{array}{cc}
35^{2} & 0 \\
0 & 35^{2}
\end{array}\right] .
$$

Figure 2A depicts the bivariate normal distributions and Figure 2B depicts corresponding equal likelihood contours. The contours were generated by slicing the two distributions horizontally at a height (likelihood) of .000057, where the contours are tangent to the optimal decision boundary. The predicted performance associated with this optimal boundary is $90 \%$ correct; that is, if a classifier responds "A" to stimuli that fall above the boundary and "B" to stimuli that fall below the boundary, then this classifier is expected to respond correctly to approximately $90 \%$ of the stimuli.

Using the category distributions and optimal decision boundary depicted in Figure 2, an experimenter may generate stimuli belonging to these predefined categories. The stimuli are generated by randomly sampling from the multivariate normal category distributions. ${ }^{2}$ Figure 3 illustrates a random sample of 500 stimuli. Two hundred fifty "+" symbols represent Category A stimuli and 250 " $\square$ " symbols represent Category B stimuli. Again, the sample of points in Figure 3 is merely a mathematical representation of the stimulus set. In reality, the participant in an experiment views a series of two-dimensional patterns, such as the circle-spoke stimuli in Figure 1, that correspond to the points. Finally, the diagonal line represents the optimal decision boundary. It is clear that some B stimuli (" $\square$ " symbols) fall above the boundary and that some A stimuli ("+" symbols) fall below the boundary. This is the case for approximately $10 \%$ of the stimuli, which explains the expected optimal performance of $90 \%$ correct.
Figure 4 illustrates hypothetical observer data. Here the "+" and "O" symbols represent the observer's category "A" and " $\mathrm{B}$ " responses rather than the true category labels. This participant responded correctly to $86.4 \%$ of the stimuli. The figure also illustrates two decision boundaries; the solid diagonal line represents the optimal boundary, and the dotted diagonal line represents the linear boundary that best separates the categorical responses.

By studying various participant boundaries using the GRT framework, the experimenter may learn about human categorization biases, strategies, and limitations. For example, if the boundary that best fits the response data is linear and parallel to the horizontal axis, then the researcher may conclude that the participant ignored (or was unable to process) stimulus information that was represented by the horizontal axis. Alternatively, if the bestfitting boundary is parallel to but shifted away from the optimal categorization rule, then the researcher may conclude that the participant favored a particular categorical response. Furthermore, by examining model-fit measures (such as the log likelihood or Akaike's [1974] information criterion provided by the GRT toolbox), the researcher may conclude that the participant was using an optimal, linear, quadratic, or other form of categorical decision boundary. As the author has discovered in her own research, this ability to examine participant decision boundaries is particularly valuable when participants are unable to verbally describe their categorization strategies or when their verbal descriptions differ from those revealed by a model-fitting analysis.

\section{GRT Toolbox}

The typical user of the GRT toolbox designs experiments for two-category tasks in which the categories are specified by multivariate normal distributions. The toolbox provides routines to help an experimenter design categorization (or identification) experiments, generate stimuli for these experiments, simulate a subject's responses, analyze categorization data, and graph results. The subroutines that generate stimuli are particularly useful because once a random sample has been generated, the sample may be transformed to make the sample parameters (means and covariances) match the population parameters. The simulation routines can help the experimenter determine the minimal number of stimuli needed to achieve informative results before running an experiment. The functions for analyzing data range from simple subroutines that compute percentage correct to more complex routines that compute $d^{\prime}$ or perform model fits.

Although an infinite number of decision boundaries may be fit to any data set, this toolbox provides routines for fitting the most popular ones, the general linear classifier (GLC) and the general quadratic classifier (GQC). Individual researchers may extend the toolbox by duplicating and modifying the GLC or GQC model-fit routines; thus the users may personalize the toolbox to serve their individual needs. Several subroutines target a specific type of data set that involves one-, two-, or three-dimensional 


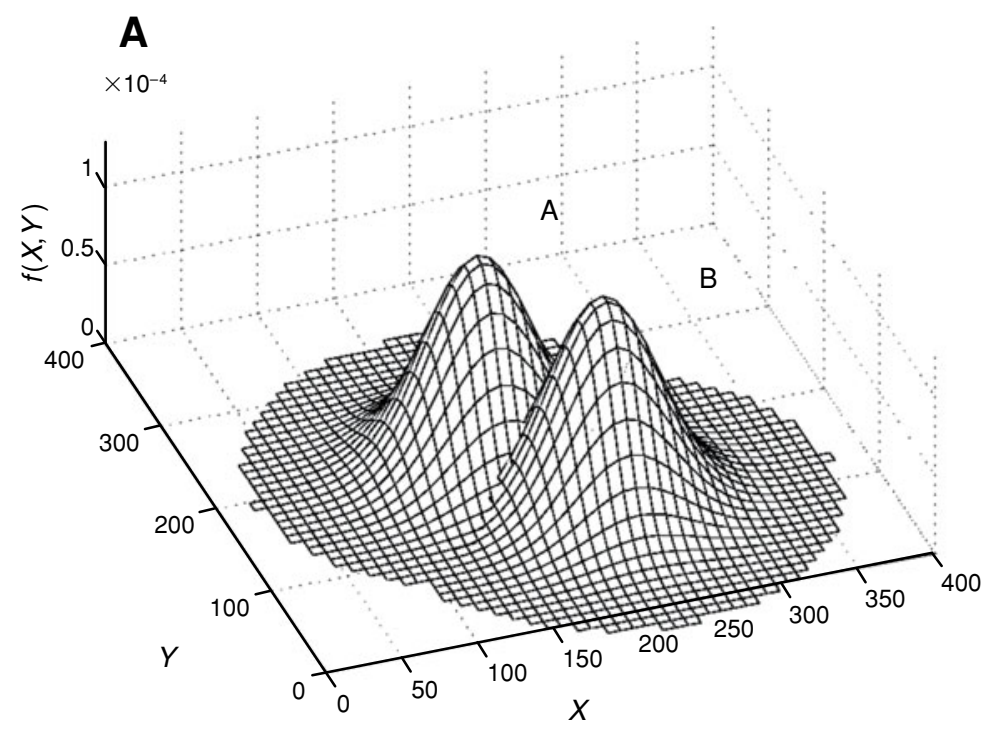

B

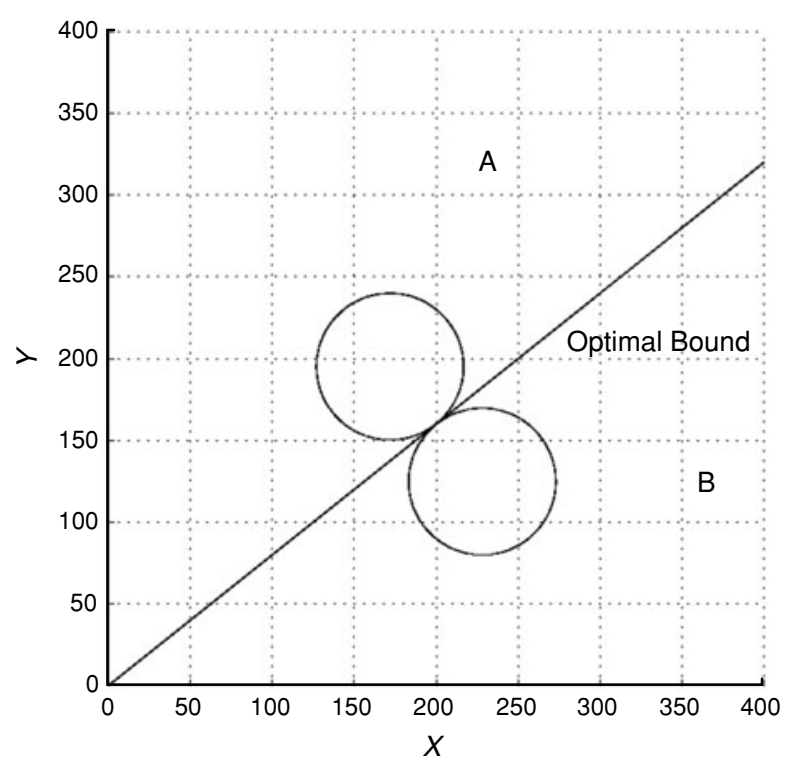

Figure 2. Two representations of bivariate normal distribution functions. (A) Bivariate normal distributions. (B) Equal likelihood contours of the bivariate normal distributions.

stimuli; however, many others operate on $n$-dimensional data. The graphics routines are handy for generating histograms of one-dimensional data and decision boundaries (lines, curves, planes, and quadratic surfaces) for two- and three-dimensional data.

Accessing and installing the software. The GRT toolbox may be downloaded from the author's Web site at rohan.sdsu.edu/ leola/toolbox.html. The software runs in MATLAB (which stands for Matrix Laboratory), a rich programming environment that is used by mathematicians, engineers, and scientists. Some data analysis functions in the GRT toolbox require the optimization toolbox. MAT$\mathrm{LAB}$ and the optimization toolbox may be purchased from
The MathWorks. Although the GRT toolbox was designed for Macintosh users, it also functions well on PCs.

The GRT toolbox consists of a folder called GRT that contains 74 subroutines and a subfolder called GRTdemos that contains 17 demo scripts. The subroutines and demo scripts are listed in Appendixes A and B.

The entire GRT folder (containing the GRTdemos subfolder) should be placed with other toolboxes in MATLAB's toolbox folder. Finally, the GRT folder and its demo subfolder must be added to the MATLAB path.

After installation. For a brief description of all the functions in the GRT toolbox, type "help GRT" in the MATLAB command window. For a brief description of 


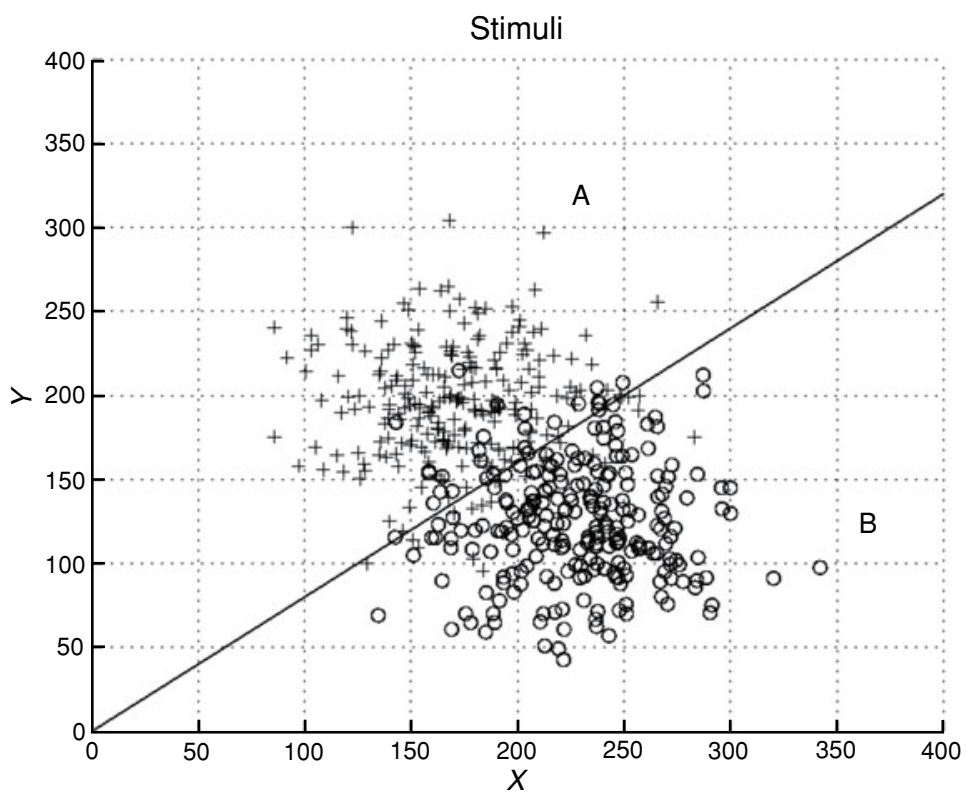

Figure 3. Representation of 500 two-dimensional stimuli with the optimal decision boundary.

Hypothetical Participant Responses

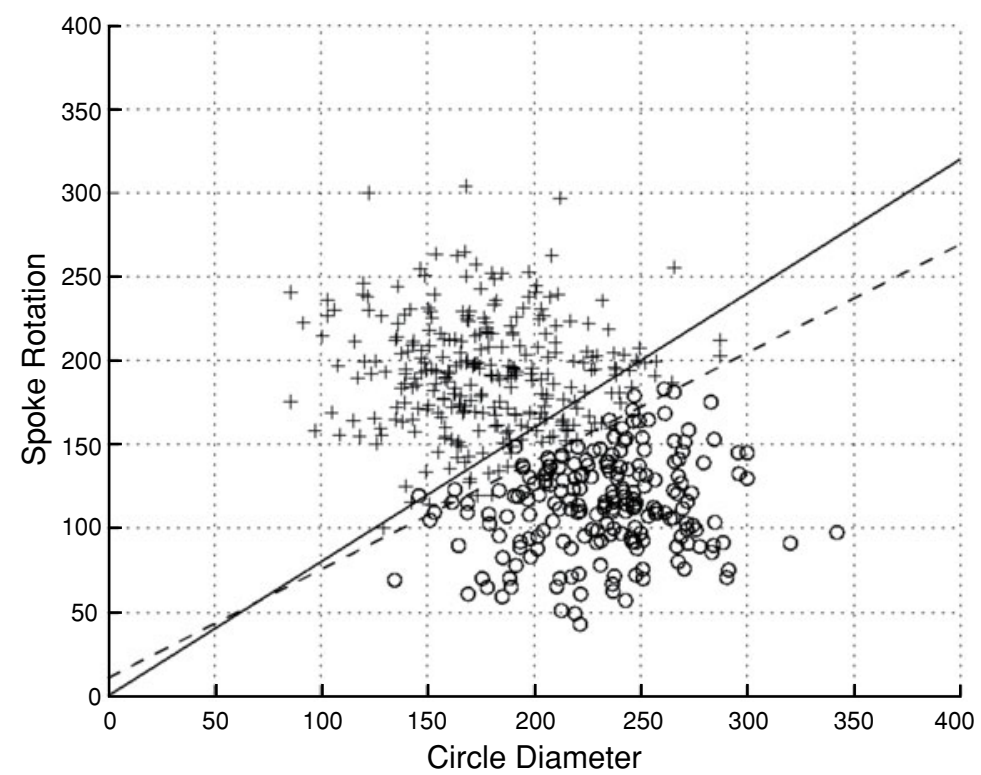

Figure 4. Representation of a hypothetical participant's category responses.

the demonstrations, type "help GRTdemos." For a description of each GRT function, type "help" followed by the function name, e.g., "help dprime." To view the contents of a function name or demonstration file, type the word "type" followed by the function or demo name, e.g., "type Comp2dStats."

An easy way to quickly become familiar with the software is to run the demos in the order in which they are listed when you type "help GRTdemos." These are the templates that the author used to generate all of the figures in this article, except Figure 1.

\section{REFERENCES}

AKaIKE, H. (1974). A new look at the statistical model identification. IEEE Transactions on Automatic Control, 19, 716-723.

Alfonso-Reese, L. A., Ashby, F. G., \& Brainard, D. H. (2002). What makes a categorization task difficult? Perception \& Psychophysics, 64, 570-583. 
AshBY, F. G. (1992). Multidimensional models of categorization. In F. G. Ashby (Ed.), Multidimensional models of perception and cognition (pp. 449-483). Hillsdale, NJ: Erlbaum.

Ashby, F. G., Alfonso-Reese, L. A., Turken, A. U., \& Waldron, E. M. (1998). A neuropsychological theory of multiple systems in category learning. Psychological Review, 105, 442-481.

Ashby, F. G., \& GotT, R. E. (1988). Decision rules in the perception and categorization of multidimensional stimuli. Journal of Experimental Psychology: Learning, Memory, \& Cognition, 14, 33-53.

AshbY, F. G., \& MADDOX, W. T. (1990). Integrating information from separable psychological dimensions. Journal of Experimental Psychology: Human Perception \& Performance, 16, 598-612.

Ashby, F. G., \& Townsend, J. T. (1986). Varieties of perceptual independence. Psychological Review, 93, 154-179.

Green, D. M., \& Swets, J. A. (1966). Signal detection theory and psychophysics. New York: Wiley.

JoHnson, R. A., \& Wichern, D. W. (2002). Applied multivariate statistical analysis (5th ed.). Upper Saddle River, NJ: Prentice Hall.

KADLEC, H. (1995). Multidimensional signal detection analyses (MSDA) for testing separability and independence: A Pascal program. Behavior Research Methods, Instruments, \& Computers, 27, 442-458.

Maddox, W. T., \& DodD, J. L. (2003). Separating perceptual and decisional attention processes in the identification and categorization of integral-dimension stimuli. Journal of Experimental Psychology: Learning, Memory, \& Cognition, 29, 467-480.
Maddox, W. T., \& ING, A. D. (2005). Delayed feedback disrupts the procedural-learning system but not the hypothesis-testing system in perceptual category learning. Journal of Experimental Psychology: Learning, Memory, \& Cognition, 31, 100-107.

Nosofsky, R. M., \& PALMERI, T. J. (1997). An exemplar-based random walk model of speeded classification. Psychological Review, 104, 266-300.

Thomas, R. D. (1998). Learning correlations in categorization tasks using large, ill-defined categories. Journal of Experimental Psychology: Learning, Memory, \& Cognition, 24, 119-143.

Thurstone, L. L. (1927). A law of comparative judgment. Psychological Review, 34, 273-286.

\section{NOTES}

1. For readers interested in software for analyzing identification data based on the general recognition theory, see Kadlec (1995).

2. This method of generating stimuli is well established among categorization researchers (e.g., Ashby et al., 1998; Ashby \& Gott, 1988; Maddox \& Ing, 2005; Thomas, 1998). It is based on the general recognition randomization technique, where "the unobservable perceptual variability postulated by the general recognition theory is replaced by external stimulus variability controlled by the experimenter" (Ashby \& Gott, 1988, p. 51).

\begin{tabular}{llll} 
& \multicolumn{2}{c}{ APPENDIXA } & \\
\hline angvec2xymat.m & gensample.m & norm_old_3dparams.m & plot3dquadbnd.m \\
bivnormpdf.m & getbndendpts.m & normalcdf.m & plot3dresp.m \\
bnd2slint.m & getcovs.m & normalcdfinv.m & plot3drespcustom.m \\
clip_vals.m & getmeans.m & normalpdf.m & plot3dstim.m \\
colorstr2colorval.m & lindecisbnd.m & old2new_2dparams.m & plotbivnormcon.m \\
colorval2colorstr.m & lindiscrim1dvals.m & old2new_3dparams.m & quadbndpercorr.m \\
dprime.m & lindiscrim2dvals.m & percorr.m & quaddecisbnd.m \\
dprimef.m & lindiscrim3dvals.m & plot1dlinbnd.m & randrows.m \\
fastnormalcdf.m & linprobcorr.m & plot1dresp.m & reducemat.m \\
fisherdiscrim1d.m & negloglike_1dGLC.m & plot1dstim.m & reducevec.m \\
fisherdiscrim2d.m & negloglike_2dGLC.m & plot2bivnorms.m & sim1dlin.m \\
fisherdiscrim3d.m & negloglike_2dGQC.m & plot2dlinbnd.m & sim2dlin.m \\
fit_1dGLC.m & negloglike_3dGLC.m & plot2dquadbnd.m & sim3dlin.m \\
fit_2dGLC.m & new2old_2dparams.m & plot2drespcustom.m & transample.m \\
fit_2dGQC.m & new2old_3dparams.m & plot2dstim.m & xymat2angvec.m \\
fit_3dGLC.m & norm_old_1dparams.m & plot2dstimcustom.m & xyzmat2angmat.m \\
\hline
\end{tabular}

APPENDIX B

Demo Scripts Available in the GRT Toolbox

\begin{tabular}{llll}
\hline Comp2dStats.m & Find3dGLC.m & Plot1dGRTdata.m & Sim2dSubj.m \\
Design2dGRTexp.m & Find3dGQC.m & Plot2BVNs.m & Sim3dSubj.m \\
Find1dGLC.m & Gen1dGRTstim.m & Plot2dGRTdata.m & \\
Find2dGLC.m & Gen2dGRTstim.m & Plot3dGRTdata.m & \\
Find2dGQC.m & Gen3dGRTstim.m & Sim1dSubj.m & \\
\hline
\end{tabular}

(Manuscript received March 24, 2005;

revision accepted for publication August 19, 2005.) 